\title{
Transient quantum transport simulation based on the statistical density matrix
}

\author{
Hiroshi Mizuta† and Chris J Goodings $\ddagger$ \\ $\uparrow$ Hitachi Cambridge Laboratory, Hitachi Europe Ltd, Cambridge CB3 OHE, UK \\ * Microelectronics Research Centre, University of Cambridge, Cambridge CB3 OHE, \\ UK
}

Received 12 October 1990

\begin{abstract}
A new quanturn device simulation technology has been developed based on the statistical density matrix theory. By introducing the Hartree self-consistent-field model for electron-electron interactions and the relaxation time approximation for scattering processes, the one-dimensional time-dependent Liouville-von Neumann equation for the electron density matrix has been solved. The density matrix is a wavefunction-wavefunction correlation function with its off-diagonal elements directly measuring the phase coherence of wavefunctions in quantum mechanical devices. We report a numerical model for the transient density matrix, calculated initially in thermal equilibrium by self-consistently solving the Schrödinger equation and Poisson's equation. The present simulation technology is applied to the simple AlGaAs/GaAs resonant tunnelling diode and the femtosecond time evolution of the density matrix under an applied field is demonstrated. Remarkable oscillatory behaviour observed in the off-diagonal elements reveals the existence of longrange phase correlations of the electron wave in the resonance state. Intrinsic bistability and switching characteristics of the device are investigated from the viewpoint of charge accumulation in the quantum well, and the peak-to-valley current ratio is discussed in terms of phase coherence degradation of electron waves due to scattering.
\end{abstract}

\section{Introduction}

Recent rapid advances in crystal growth and microfabrication technologies have allowed us to explore a new field of semiconductor device research. The quantum mechanical wave nature of electrons is in evidence in mesoscopic semiconductor structures with sizes below $100 \mathrm{~nm}$. Instead of conventional devices such as field-effect transistors and bipolar transistors, which can be described by the classical particle model, a variety of novel device concepts have been proposed based on the quantum mechanical features of carriers. One pioneering device in this field is the resonant tunnelling diode (RTD) [14] which utilizes the electron wave resonance in finite superlattices. Negative differential resistance obtained in these structures leads to multistable device operation, and several new devices have been successfully developed. Following this, various other types of quantum mechanical devices have been proposed, the quantum interference transistor [5], the quantum diffraction field-effect transistor [6], and the quantum-modulated transistor [7] being typical ideas that use electron wave interference phenomena.

At the same time, theoretical reserch on very small semiconductor devices is also at the threshold of the quantum mechanical field. Although classical ballistic transport 
modelling is very significant for present deep-submicrometre devices, an advanced simulation technology based on the quantum transport theory is certainly going to be required to parallel the improvement in nanometre-scale fabrication technology. Preliminary quantum mechanical modelling based on the Schrödinger equation follows the so-called scattering model which, because of its simplicity, has often been used to analyse RTDS [8,9], quantum wires [10] and the quantum-modulated transistor [7]. One major problem of this model is the fact that we have to assume the statistical distribution of carriers in advance. Systems are usually treated as perfect conductors with no dissipation process, so this model can be applied only to completely ballistic systems close to thermal equilibrium. In addition, except for in simple resonant tunnelling diodes, self-consistency of the electrostatic potential is often neglected because of the uncertainty in the carrier distribution. In order to simulate the statistical distribution of carriers as well as quantum mechanical states, it is obviously necessary to solve the quantum transport equation rather than the Schrödinger equation. In the quantum transport theory several formulations have been reported for quantum device simulation, such as the density matrix [11], the Wigner function [12], Green functions [13], and path integrals [14]. Very recently, the quantum mechanical Wigner function has been solved numerically [15-18] to analyse electron transport in RTDS. The equation of motion of the Wigner function, which is derived from the Liouville-von Neumann equation for the statistical density matrix by applying the Wigner-Weyl transformation, has been solved in the finite-difference scheme, and some calculated results have been reported on the steady-state $I-V$ characteristics and transient behaviour of RTDS. Although some major problems such as boundary conditions and correct initial conditions for the Wigner function are still under discussion, the successful application of the Wigner function formalism to electron devices is certainly a significant advance in quantum device simulation technology.

The purpose of this work is to present a new quantum device simulation technology based upon the density matrix equation [11] which is the most fundamental equation in statistical quantum mechanical physics. The statistical density matrix is a wavefunctionwavefunction correlation function, and its off-diagonal elements directly measure the phase coherence of wavefunctions: one of the most significant parameters in electron wave device design. The first numerical calculation of the density matrix equation was reported by Frensley [19], who observed for the first time the time evolution of the density matrix for the resonant tunnelling diode. At the same time, however, a lot of problems were pointed out in this formalism, such as Ohmic contact boundary conditions, a correct initial density matrix, a self-consistent Hartree potential, and numerical instability in transient calculations. Very recently, a Monte Carlo calculation to simulate the effect of electron-phonon interaction on the density matrix was also reported [20-22]. In the present paper, we report a numerical quantum device model based on the direct calculation of the density matrix equation, which includes new boundary and initial conditions for the density matrix and an implicit scheme for time integration. The correct density matrix in thermal equilibrium is calculated by solving the Schrödinger equation and Poisson's equation self-consistently. Using the thermal equilibrium density matrix as an initial state, the time-dependent density matrix equation is then solved directly. The Hartree self-consistent field model and the relaxation time approximation are introduced for electron-electron interactions and scattering processes, respectively. The present simulation technology is applied to the simple $\mathrm{AlGaAs} / \mathrm{GaAs}$ resonant tunnelling diodes, and transient behaviours of electron buildup in the quantum well and development of long-range phase correlations between 
quasi-bound electron waves and free electron waves are demonstrated. In addition, the switching behaviour from the peak (valley) to the valley (peak) of the $I-V$ characteristics and the effects of scattering on the peak-to-valley current ratio are discussed.

\section{Thermal equilibrium density matrix}

The first step of the quantum device simulation is to find the correct statistical density matrix for a system in thermal equilibrium, which is used as an initial condition for the time-dependent density matrix equation. Provided that an unperturbed quantum mechanical open system is in thermal equiibrium with its surroundings, the statistical density matrix based on the independent-particle approximation is expressed by the following equation:

$$
\rho_{0}\left(x, x^{\prime}\right)=\left\langle\Psi(x) \Psi^{*}\left(x^{\prime}\right)\right\rangle=\sum_{k, \sigma} \Psi_{k}(x) \Psi_{k}^{*}\left(x^{\prime}\right) f(k)
$$

where $\Psi_{k}(x)$ is the eigenfunction of the unperturbed Hamiltonian, and $f(k)$ is the FermiDirac distribution function. Equation (1) shows that the statistical density matrix is a function of the two positions $\boldsymbol{x}$ and $\boldsymbol{x}^{\prime}$ : the diagonal elements represent the real electron density in the system and the off-diagonal elements measure the phase correlations of the wavefunctions. A complete set of wavefunctions is obtained by solving the timeindependent effective-mass Schrödinger equation:

$$
H \Psi_{k}(x)=-\frac{\hbar^{2}}{2} \nabla\left(\frac{1}{m^{*}(x)} \nabla\right) \Psi_{k}(x)+V(x) \Psi_{k}(x)=E_{k} \Psi_{k}(x)
$$

where $m^{*}(x)$ is the electron effective mass and $V(x)$ is the potential energy distribution which consists of the electron affinity, $V_{0}(x)$, and the Hartree potential, $V_{\mathrm{SC}}(x)$, due to the charge density of electrons and ionized donors. The Hartree potential is determined self-consistently by solving Poisson's equation:

$$
-\nabla^{2} V_{\mathrm{SC}}(x)=\frac{1}{\varepsilon(x)}\left(N_{\mathrm{D}}^{+}(x)-\sum_{k, \sigma}\left|\Psi_{k}(x)\right|^{2} f(k)\right) .
$$

The Schrödinger equation (2) is solved by using the finite-difference method rather than the transfer matrix method [9]. From the point of view of numerical calculations, the transfer matrix method is more accurate than the finite-difference method because wavefunctions between mesh point are stored as plane-wave states. However, the use of the solutions obtained by the transfer matrix method sometimes brings about an unrealistic current density at hetero-interfaces when the resultant density matrix is used as an initial solution for the time-dependent density matrix equation. This is because of the difference between the ways in which the Schrödinger equation and the density matrix equation are discretized. In the present work, we adopt exactly the same finitedifference discretization for both equations.

A set of incoming scattering states (cathode- and anode-incident plane waves) [23] are used as boundary conditions to solve the Schrödinger equation. When the finitedifference method is used for solving the Schrödinger equation, solutions are no longer expressed as coefficients of plane waves. It is therefore necessary to decompose the wavefunctions obtained at the edge of devices into incident and reflected plane waves to find a normalization factor. From the unnormalized solutions on the first two mesh 
points, we evaluate the coefficients for the incident and reflected waves and normalize the wavefunctions. Accuracy of this normalization method depends on the first mesh spacing and, to some extent, can be judged from the resulting electron density calculated at the cathode and anode edge.

In the present paper, we adopt the simple structure of an AIGaAs/GaAs doublebarrier resonant tunnelling diode to examine the new simulation technology. The assumed structure consists of a undoped GaAs quantum well $5 \mathrm{~nm}$ thick, two undoped $\mathrm{Al}_{0.33} \mathrm{Ga}_{0.67} \mathrm{As}$ barriers $4 \mathrm{~nm}$ thick, and highly doped $n$-type GaAs $\left(N_{\mathrm{D}}=1 \times 10^{18} \mathrm{~cm}^{-3}\right)$ cathode and anode layers $20 \mathrm{~nm}$ thick with undoped GaAs spacer layers $6 \mathrm{~nm}$ thick. The conduction band discontinuity in the GaAs/AlGaAs heterostructure is assumed to be $60 \%$ of the $\Gamma$-band energy gap difference. Positional dependence of electron effective mass, $m^{*}(x)$, is taken into account by assuming the simple Al mole fraction dependence of the electron mass in AlGaAs: $m^{*}(\mathrm{GaAs})=0.067 m_{0}$ and $m^{*}\left(\mathrm{Al}_{0.33} \mathrm{Ga}_{0.67} \mathrm{As}\right)=$ $0.094 m_{0}$. Figure $1(a)$ shows the real parts of the equilibrium density matrix (the imaginary parts are exactly zero), and figure $1(b)$ the energy band diagram calculated self-con-

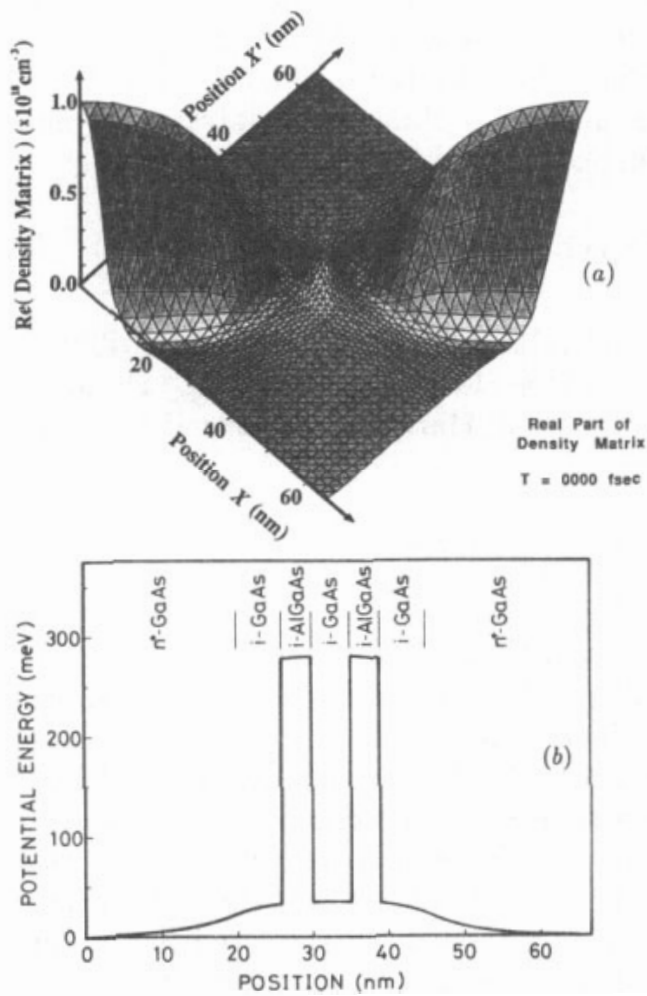

Figure 1. (a) The equilibrium density matrix and (b) energy band diagram, calculated self-consistently for the AlGaAs/GaAs as resonant tunnelling diode at a temperature of $300 \mathrm{~K}$. The transverse axis in (b) has been adjusted so that the diagonal of the density matrix, that is the electron distribution, is displayed with the same position scale as the band diagram.

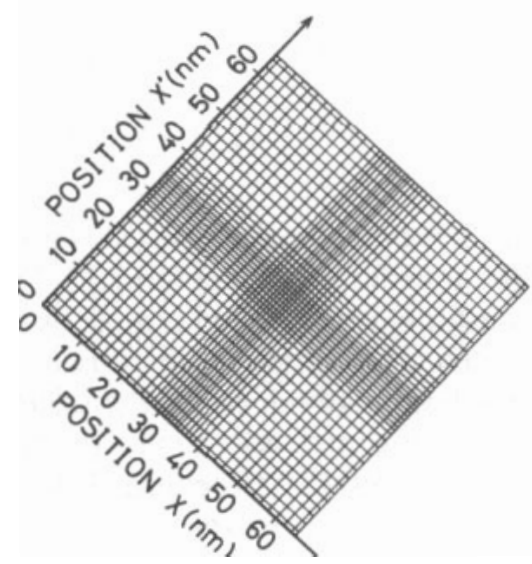

Figure 2. The position mesh grid used for the density matrix of the resonant tunnelling diode. The mesh spacing has been chosen to be small for the AIGaAs barriers and the GaAs quantum well. 
sistently at a temperature of $300 \mathrm{~K}$. An energy mesh with a spacing of $0.5 \mathrm{meV}$ was used to calculate and store all the wavefunctions of the system with eigenenergies up to $200 \mathrm{meV}$. The normalization method for wavefunctions described above appears to be successful since charge-neutral regions assured by the flat energy band are obtained near the cathode and anode edges. The small peak in the centre of figure 1(a) represents accumulated electrons in the quantum well. These electrons are thermally distributed around the first quasi-bound state (the resonant state) at an energy of about $60 \mathrm{meV}$ measured from the bottom of the quantum well. The steep decrease in electron density near the double barriers stems from a quantum repulsion of electron waves [17]. In figure $1(b)$, the upward shift of the potential diagram near the heterointerfaces in the cathode and anode layers is caused by the self-consistent field from electrons injected into the spacer layers. A typical mesh grid adopted for the density matrix of the resonant tunnelling diode is shown in figure 2 : the mesh spacing was chosen to be smaller in the region of the double-barrier structure. This sort of non-uniform mesh is useful for saving computational time and memory when solving the density matrix equation, although it complicates the coefficient matrix of the discretized equation.

As mentioned earlier, off-diagonal structures of the density matrix measure the phase correlations between the electron wavefunctions at different positions. In figure $1(a)$, the density matrix in the cathode and anode regions varies steeply along the crossdiagonal direction, and its dependence on a relative distance $x-x^{\prime}$ is approximately expressed by the following Gaussian-like analytical form for the free electron density matrix:

$$
\rho_{\mathrm{FE}}\left(x, x^{\prime}\right)=\left(1 / \sqrt{2 \pi^{3} \lambda_{\mathrm{T}}^{3}}\right) \exp \left(-\left|x-x^{\prime}\right|^{2} / 2 \lambda_{\mathrm{T}}^{2}+E_{\mathrm{F}} / k_{\mathrm{B}} T\right)
$$

where a thermal coherence length of the electron wave, $\lambda_{\mathrm{T}}$, is defined as follows:

$$
\lambda_{\mathrm{T}}^{2}=\hbar^{2} / m^{*} k_{\mathrm{B}} T \text {. }
$$

The thermal coherence length, which is about $7 \mathrm{~nm}$ for GaAs at room temperature, is a critical length in multi-mode (multi-phased) electron wave devices, and the correlation between thermally distributed free electrons decreases steeply beyond $\lambda_{\mathrm{T}}$ because of the superposition of electron waves with various phases. In section 4 , the development of dynamical interference between these free electron waves and quasi-bound electron waves at the resonant state is demonstrated.

\section{Transport theory based on the statistical density matrix}

The second step of the quantum device simulation is to solve the fundamental equation of motion for the density matrix. The equilibrium density matrix, $\rho_{0}$, obtained in the previous section is used as an initial state for the density matrix equation. The time evolution of the density matrix under an applied electric field is given by the following Liouville-von Neumann equation:

$$
\partial \rho / \partial t=(1 / \mathrm{i} \hbar)[H(t), \rho]+C_{\mathrm{col}} \rho
$$

where $H(t)$ is the Hamiltonian under an applied bias, and $C_{\mathrm{col}}$ is a collision operator, discussed later. Using the position-space representation, the density matrix equation is 
given by the following expression:

$$
\begin{aligned}
\frac{\partial \rho\left(x, x^{\prime} ; t\right)}{\partial t}= & \frac{\mathrm{i} \hbar}{2}\left[\frac{\partial}{\partial x}\left(\frac{1}{m^{*}(x)} \frac{\partial}{\partial x}\right)-\frac{\partial}{\partial x^{\prime}}\left(\frac{1}{m^{*}\left(x^{\prime}\right)} \frac{\partial}{\partial x^{\prime}}\right)\right] \rho\left(x, x^{\prime} ; t\right) \\
& +\frac{1}{\mathrm{i} \hbar}\left(V(x ; t)-V\left(x^{\prime} ; t\right)\right) \rho\left(x, x^{t} ; t\right)+C_{\mathrm{col}} \rho\left(x, x^{\prime} ; t\right) \\
V(x ; t)=V_{0}(x) & +V_{\mathrm{SC}}(x ; t)
\end{aligned}
$$

where the time-dependent Hartree potential $V_{\mathrm{SC}}(x ; t)$ is determined by the diagonal elements of the density matrix as follows:

$$
(\mathrm{d} / \mathrm{d} x)\left(\varepsilon(x) \mathrm{d} V_{\mathrm{SC}}(x ; t) / \mathrm{d} x\right)=-q\left[N_{\mathrm{D}}^{+}(x)-\operatorname{Re}(\rho(x, x ; t))\right] .
$$

The time-dependent conduction current density, $j(x ; t)$, is then calculated from the following formula:

$$
j(x ; t)=\left.\left(q \hbar / 2 m^{*}(x) \mathrm{i}\right)\left\{\left(\partial / \partial x-\partial / \partial x^{\prime}\right) \rho\left(x, x^{\prime} ; t\right)\right\}\right|_{x^{\prime}=x} .
$$

Equation (7) is discretized in $x$ and $x^{\prime}$ by using exactly the same spatial grid as the equilibrium density matrix and then deformed to algebraic equations. For the time integral of (7), an implicit scheme is used to obtain stable convergence in the numerical calculations because explicit schemes usually require extremely small time steps [19]. Since the Hartree potential depends on the density matrix (equation (9)), full implicit treatment for (7) gives rise to a complicated system of coupled non-linear equations. Here, we employ the implicit scheme only for the density matrix and treat the Hartree potential explicitly. The resultant finite-difference equation is as follows:

$$
\begin{aligned}
\frac{\rho_{i, j}(t+\Delta t)-}{\Delta t} & \rho_{i, j}(t) \\
= & c\left\{\frac { \mathrm { i } \hbar } { 2 } \left[\frac { 1 } { \Delta \tilde { x } _ { i } } \left(\frac{1}{m_{i+1 / 2}^{*}} \frac{\rho_{i+1, j}(t+\Delta t)-\rho_{i, j}(t+\Delta t)}{\Delta x_{i}}\right.\right.\right. \\
& \left.+\frac{1}{m_{i-1 / 2}^{*}} \frac{\rho_{i-1, j}(t+\Delta t)-\rho_{i, j}(t+\Delta t)}{\Delta x_{i-1}}\right) \\
& -\frac{1}{\Delta \tilde{x}_{j}}\left(\frac{1}{m_{j+1 / 2}^{*}} \frac{\rho_{i, j+1}(t+\Delta t)-\rho_{i, j}(t+\Delta t)}{\Delta x_{j}}\right. \\
& \left.\left.+\frac{1}{m_{j-1 / 2}^{*}} \frac{\rho_{i, j-1}(t+\Delta t)-\rho_{i, j}(t+\Delta t)}{\Delta x_{j-1}}\right)\right] \\
& \left.+\frac{1}{\mathrm{i} \hbar}\left(V_{i}(t)-V_{j}(t)\right) \rho_{i, j}(t+\Delta t)+\left(C_{\mathrm{col}} \rho\right)_{i, j}(t+\Delta t)\right\} \\
& +(1-c)\left\{\frac { i \hbar } { 2 } \left[\frac{1}{\Delta \tilde{x}_{i}}\left(\frac{1}{m_{i+1 / 2}^{*}} \frac{\rho_{i+1, j}(t)-\rho_{i, j}(t)}{\Delta x_{i}}+\frac{1}{m_{i-1 / 2}^{*}} \frac{\rho_{i-1, j}(t)-\rho_{i, j}(t)}{\Delta x_{i-1}}\right)\right.\right. \\
& \left.-\frac{1}{\Delta \tilde{x}_{j}}\left(\frac{1}{m_{j+1 / 2}^{*}} \frac{\rho_{i, j+1}(t)-\rho_{i, j}(t)}{\Delta x_{j}}+\frac{1}{m_{j-1 / 2}^{*}} \frac{\rho_{i, j-1}(t)-\rho_{i, j}(t)}{\Delta x_{i-1}}\right)\right] \\
& +\frac{1}{\mathrm{i} \hbar}\left(V_{i}(t)-V_{i}(t)\right) \rho_{i, j}(t) \\
& \left.+\left(C_{\mathrm{col}} \rho\right)_{i, j}(t)\right\} \quad(i, j=1,2, \ldots, N)
\end{aligned}
$$


where indices $i$ and $i+1 / 2$ represent original and half-meshpoints, $\Delta x_{i}$ and $\Delta \tilde{x}_{i}$ the distances between the original and half-meshpoints, respectively. A numerical constant, $c$, varies from zero to unity depending on the implicit scheme used: the scheme $c=\frac{1}{2}$ is a usual Crank-Nicolson-type implicit scheme, and $c=1$ is a backward Euler. The coefficient matrix of these simultaneous equations (11) is basically sparse (except for the unknown collision term) although it is not symmetric and contains complex elements. This contrasts with the fact that the coefficient matrix in the Wigner function formalism is generally dense. The discretized Wigner function requires storage proportional to $N_{x} N_{k}^{2}$ [16] where $N_{x}$ and $N_{k}$ represent numbers of meshpoints in the position and momentum spaces, while the density matrix equation only necessitates storage proportional to $N^{2}$ where $N$ represents the number of meshpoints in $x$ and $x^{\prime}$ dimensions. Thus, from the view of numerical calculation, the density matrix formalism has the great advantage of saving computational memory. By converting these $N$ complex equations into $2 \mathrm{~N}$ equations with real coefficients, equation (11) can be solved by several methods for sparse linear systems. We adopt here the conjugate gradient method for nonsymmetric sparse matrices.

The collision term, $C_{\text {col }} \rho$, which introduces dissipation processes and causes time irreversibility of the system, is a non-trivial part of the quantum transport theory. Several discussions have been reported on the collision term in the density matrix formalism. First, an accurate quantum mechanical expression for electron-phonon interaction was reported by Levinson [24]. He derived the closed equation for the density matrix of electrons which are weakly interacting with equilibrium phonons. In his formula, however, the interaction term involves time integrations (i.e. the past history of the system), for which numerical calculations are non-trivial. Second, a semi-classical model was proposed by Caldeira and Leggett [25] relating to the theory of quantum Brownian motion:

$$
\begin{aligned}
C_{\mathrm{col}} \rho\left(x, x^{\prime} ; t\right) & =-\gamma\left(x-x^{\prime}\right)\left(\partial \rho\left(x, x^{\prime} ; t\right) / \partial x-\partial \rho\left(x, x^{\prime} ; t\right) / \partial x^{\prime}\right) \\
& -\left(2 m^{*} \gamma k_{\mathrm{B}} T / \hbar^{2}\right)\left|x-x^{\prime}\right|^{2} \rho\left(x, x^{\prime} ; t\right)
\end{aligned}
$$

where $\gamma$ is the coupling constant of electrons to a reservoir system. Although the expression is simple enough to be applied to numerical simulations, it is verified that this model is only correct when the thermal energy, $k_{\mathrm{B}} T$, is much larger than the coupling energy between the electrons and the reservoir. Third, the simplest model for the collision term is a well known relaxation time approximation:

$$
C_{\mathrm{col}} \rho\left(x, x^{\prime} ; t\right)=\left(\rho\left(x, x^{\prime} ; t\right)-\rho_{\mathrm{QE}}\left(x, x^{\prime}\right)\right) / \tau_{\mathrm{s}}
$$

where $\rho_{\mathrm{QE}}$ is the quasi-equilibrium density matrix under an external bias, and $\tau_{\mathrm{s}}$ is a macroscopic relaxation time parameter which represents all of the scattering processes. In the present work, we choose the relaxation time approximation because of the simplicity it leads to in numerical calculations. One difficulty with the use of this approximation for a real device is the fact that the distribution of electrons varies with position and external bias. Equation (13) has an effect on the position-space distribution of electrons and, if the thermal equilibrium density matrix $\rho_{0}$ is used directly as $\rho_{\mathrm{QE}}$, this results in a breaking of the current continuity. Obviously, an adequate model for $\rho_{\mathrm{QE}}$ is necessary which has the same electron distribution as $\rho\left(x, x^{\prime} ; t\right)$. The following approximation for $\rho_{\mathrm{QE}}$ has been proposed by Frensley [19] assuming Boltzmann statistics for 
electrons:

$$
\rho_{\mathrm{OE}}\left(x, x^{\prime} ; t\right)=\sqrt{\rho(x, x ; t) \rho\left(x^{\prime}, x^{\prime} ; t\right)} \exp \left(-\left|x-x^{\prime}\right|^{2} / \dot{\lambda}_{\mathrm{T}}^{2}\right) .
$$

This expression is easily found to be correct for the thermally distributed free electrons, but its general justification might relay on the fact that (14) reproduces the correct thermal equilibrium density matrix given by (1) [19]. In the present work, we adapt (14) for $\rho_{\mathrm{OE}}$ by adding a numerical correction for the difference between Boltzmann and Fermi-Dirac distributions. The resultant expression gives us a quantitatively correct reproduction of off-diagonal elements of $\rho_{0}$ in thermal equilibrium.

The Ohmic contact boundary condition for the density matrix is also an active field of study in the quantum transport theory in connection with the time irreversibility of quantum devices. The density matrix elements on boundaries represent correlations between wavefunctions at an electrode and at all other points in the device. Obviously, Dirichlet-type boundary conditions cannot be used on the density matrix because this would mean that the quantum current density at the Ohmic contact would be fixed from the beginning (see (10)). This fact is one of the big problems of the density matrix method in contrast to the Wigner function formalism where the so-called time-irreversible boundary condition $[16,26]$ has been proposed. One possible solution is to use Neumann-type boundary conditions. Frensley $[19,26]$ proposed the following special boundary condition:

$$
\left.\left(\partial / \partial x+\partial / \partial x^{\prime}\right) \rho\left(x, x^{\prime} ; t\right)\right|_{\text {bourdary }}=0
$$

for the density matrix taking account of the symmetry of the density matrix for thermally distributed free electrons (see (4)). From the physical point of view, this boundary condition models a black-body contact which absorbs incoming electron waves. The boundary condition (15) conserves electron density at the device edge when external bias is applied to the device. Thus, it is suitable to describe the boundary of the so-called Ohmic region near electrodes where free electrons are thermally distributed. This boundary condition, however, cannot be used for the whole boundary because it is correct only when the system has at least approximately translational invariance. For example, it is successfully applied to simple structures such as $n^{+}-n^{-}-n^{+}$diodes where the electron wavefunctions are spatially spread over the system, and the wavenumber $k$ is good quantum number. Typical quantum device structures, however, such as finite superlattices and quantum wells make (quasi-) bound electron states and are no longer translationally invariant except in the Ohmic regions near the cathode and anode electrodes. The correlations between those localized electrons and free electrons in the cathode and anode layers are long range, and the density matrix varies along $x$ and $x^{\prime}$ directions rather than along the cross-diagonal direction. In these cases, therefore, the following Neumann boundary conditions

$$
\partial \rho\left(x, x^{\prime} ; t\right) /\left.\partial x\right|_{x=0, L}=0 \quad \partial \rho\left(x, x^{\prime} ; t\right) /\left.\partial x^{\prime}\right|_{x^{\prime}=0, L}=0
$$

are plausible, rather than the boundary condition (15). These boundary conditions physically mean that there is no source at the electrodes to have an influence on the correlation function within the device. In the present work, we divide the system into two parts: the Ohmic region which is now defined as the $n^{+}$-GaAs region of thickness $20 \mathrm{~nm}$, and the essential double-barrier region (with spacer layers) causing quantum mechanical effects. The boundary condition (15) is used only for the boundaries of the Ohmic regions, and those of the active region are treated by using (16). 


\section{Calculated results and discussion}

\subsection{Transient behaviour of the density matrix}

In this section the transient behaviour of the resonant tunnelling diode under an applied bias is simulated numerically by solving the discretized density matrix equation (11). The equilibrium density matrix obtained in section 2 is used as an initial condition, and the spatial mesh grid shown in figure 2 is adopted. The new Neumann-type boundary condition described in the previous section is implemented to solve the density matrix equation. In the present calculations values of 1.0 to $2.0 \mathrm{fs}$ are used for the time step, $\Delta t$, which are small enough to represent the transient response of the resonant tunnelling diode. The relaxation time parameter, $\tau_{\mathrm{s}}$, due to scattering is typically set to be $100 \mathrm{fs}$, which corresponds to a mobility of $2600 \mathrm{~cm}^{2} \mathrm{~V}^{-1} \mathrm{~s}^{-1}$ for $\mathrm{n}-\mathrm{GaAs}$ with a donor concentration of $1 \times 10^{18} \mathrm{~cm}^{-3}$ at room temperature. The density matrix, potential profile, and current density calculated by using (10) are monitored to check the convergence of the system to a steady state. The correctness of the thermal equilibrium density matrix as an initial condition is continually checked by solving the density matrix equation under zero applied voltage. The maximum errors in the potential profile and current density under zero bias are less than $1.0 \mathrm{meV}$ and $1.0 \mathrm{~A} \mathrm{~cm}^{-2}$ at a time of $1500 \mathrm{fs}$ respectively. These are small enough to assure the suitability of the evaluated values under an applied voltage. The transient calculation has been done on a DECsystem 5810 (18.7 MIPS machine) and usually required approximately 180 minutes of CPU time to reach a steady state.

The calculated transient response of the density matrix for the resonant tunnelling diode is shown in figures 3 and 4 . These figures show the real (figure 3 ) and imaginary (figure 4) parts of the density matrix for several times when an external voltage of $120 \mathrm{mV}$, corresponding to the peak voltage of the device, is suddenly applied at $t=0$ : (a) at $t=50 \mathrm{fs} ;(b)$ at $t=100 \mathrm{fs} ;(c)$ at $t=200 \mathrm{fs} ;(d)$ at $t=600 \mathrm{fs}$; and $(e)$ at $t=1000 \mathrm{fs}$. The system reaches the steady state at a time of about $1000 \mathrm{fs}$, and no numerical instability [19] was observed. This is presumably because of improvements in the present model such as the self-consistent Hartree field, a correct initial condition, the new boundary condition, and the implicit scheme for time integration. The calculated density matrix enables us to observe not only the time-dependent variation of the electron distribution but also that of the electron correlations in the system. The diagonal of the real parts shows time dependence of the electron build-up in the quantum well as well as that of electron accumulation in the cathode region and depletion in the anode region. In the steady state, the peak concentration of electrons in the quantum well amounts to $2.5 \times 10^{17} \mathrm{~cm}^{-3}$. In figures 3 and 4 , three major features should be noticed on the offdiagonal elements of the density matrix. The large cross-diagonal structure (indicated by point $\mathrm{A}$ in figure $4(a)$ ) in the imaginary parts represents short-range phase correlations, which cause plasma current oscillations. If the momentum-space representation is used instead of the position-space representation, it is equivalent to the centre of the distribution function oscillating along the momentum axis. In other words, electrons are in collective motion in the cathode and anode regions. This phenomenon can be also observed as an oscillation of real electron density (the diagonal of figure 3 ). The most remarkable feature is an oscillatory behaviour of the imaginary parts (indicated by point $B$ in figure $4(e)$ ). It should be noted that this extends from the centre to the edges of the domain. It is caused by a quantum interference between the quasi-bound electron waves at the resonant state and free electron waves in the cathode and anode layers. We believe this to be the first direct observation of the dynamical correlations of the electron waves 

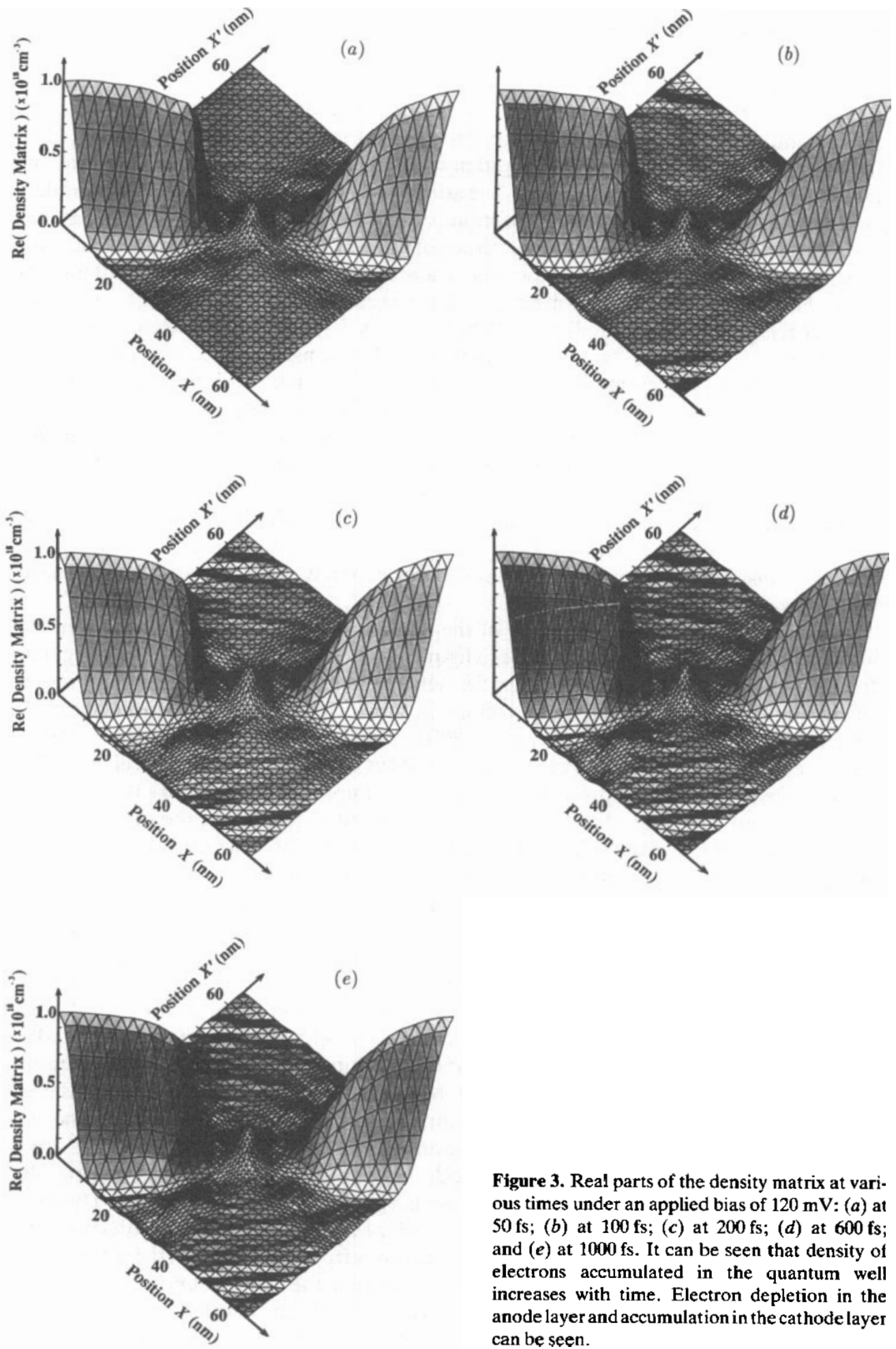

Figure 3. Real parts of the density matrix at various times under an applied bias of $120 \mathrm{mV}:(a)$ at $50 \mathrm{fs}$; (b) at $100 \mathrm{fs}$; (c) at $200 \mathrm{fs} ;(d)$ at $600 \mathrm{fs}$; and (e) at $1000 \mathrm{fs}$. It can be seen that density of electrons accumulated in the quantum well increases with time. Electron depletion in the anode layer and accumulation in the cat hode layer can be seen. 

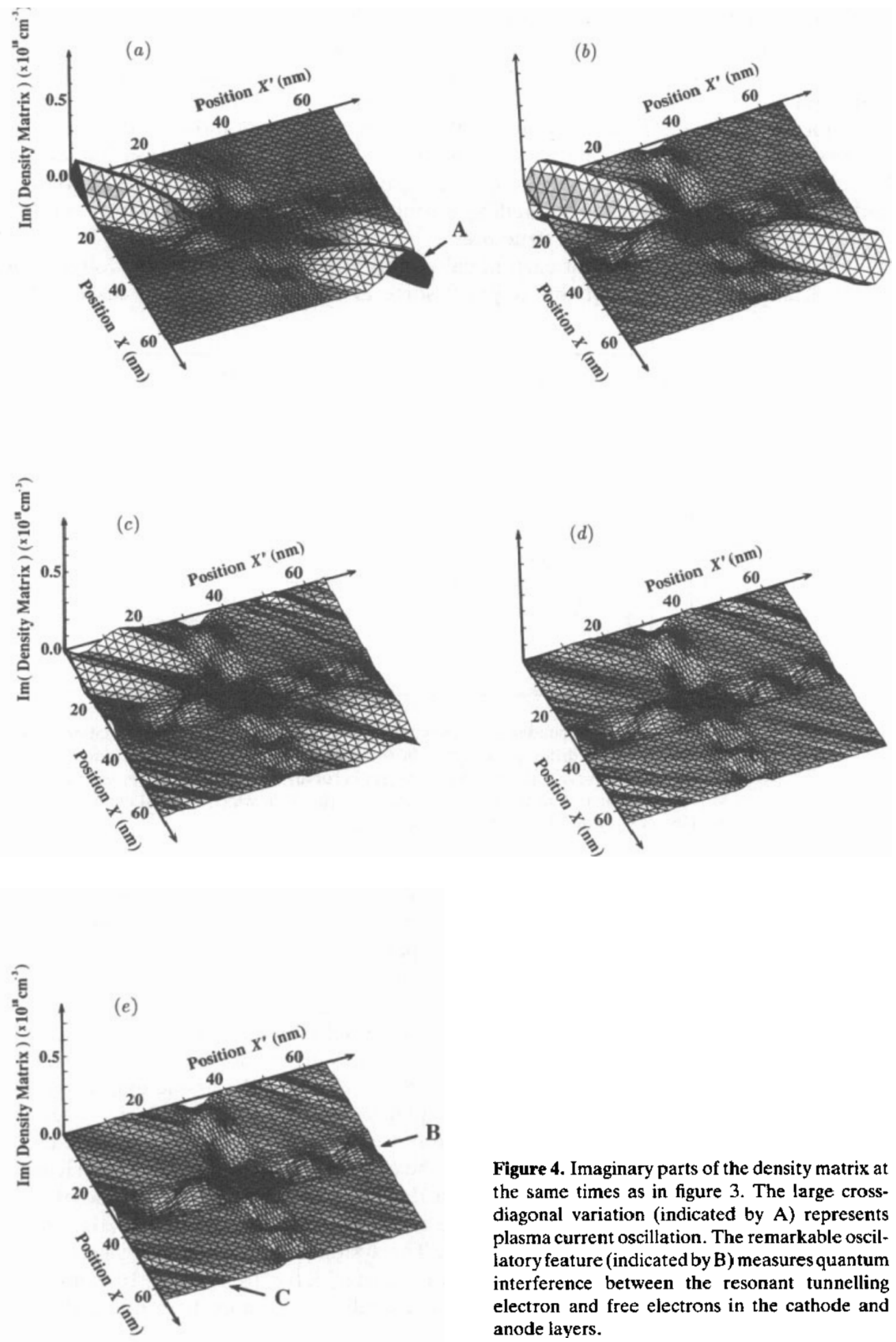

Figure 4. Imaginary parts of the density matrix at the same times as in figure 3 . The large crossdiagonal variation (indicated by $A$ ) represents plasma current oscillation. The remarkable oscillatory feature (indicated by $B$ ) measures quantum interference between the resonant tunnelling. electron and free electrons in the cathode and anode layers. 
in the resonant tunnelling diode under an applied voltage. The calculated results reveal that the electron waves at the resonant state correlate with free electron waves over the whole device. In other words, the phase coherence of the resonant electron waves is still maintained although they suffer degradation of coherence due to scattering. The relation between amplitude of the oscillation and the scattering relaxation time is discussed in the next section. Further oscillatory behaviour along the diagonal direction (indicated by point $C$ in figure $4(e)$ ) represents correlations between free electrons in the cathode and anode regions by normal tunnelling through the double barriers, which can be observed even in a single-barrier structure.

The corresponding transient current calculated by using equation (10) is shown in figure 5, and the meaning of this requires some explanation. Strictly speaking, the

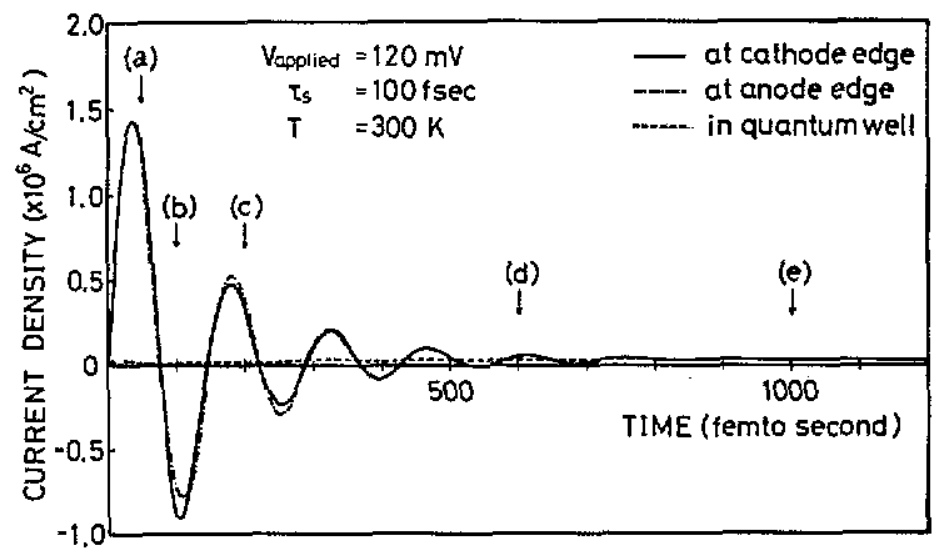

Figure 5. The time dependence of the current density calculated by using equation (10). Current densities at different positions in the device are monitored versus time: at the cathode edge (full curve); at the anode edge (chain curve); and in the quantum well (broken curve). The five arrows in the $(a)-(e)$ indicate the times for which the real and imaginary parts of the density matrix are shown in figure 3 and 4.

current density defined by equation (10) is correct only in the steady state since we did not include the time-dependent displacement current. Because of this simplification, the transient current density has a positional dependence in figure 5: the current density at the cathode edge, the anode edge, and in the quantum well are shown by a full curve, a chain curve, and a broken curve, respectively. Although it is possible to calculate the displacement current from the time derivative of the self-consistent Hartree potential, we intentionally show only the time dependence of the current caused by the change in electron density. The current density at the cathode edge (or the anode edge) can be regarded as the total current because the displacement current at the device edge is nearly zero. As described above, large current oscillation is observed in figure 5 as a result of a plasma-type motion of electrons in the cathode and anode regions, which was seen in the imaginary parts of the density matrix (figures $4(a)-(e)$ ). The period of the current oscillation of about $150 \mathrm{fs}$ is mainly determined by the density and effective mass of electrons in the cathode and anode layer. The system reaches the steady state at a time of about $1000 \mathrm{fs}$, resulting in the steady current of $3.1 \times 10^{4} \mathrm{~A} \mathrm{~cm}^{-2}$. It should be noted that the first peak current of the plasma oscillation is more than one order of magnitude larger than the steady current. 


\subsection{I-V characteristics and switching behaviour}

The steady-state $I-V$ characteristics are obtained for the above transient calculations. First, a small external voltage, which is typically 5 to $10 \mathrm{mV}$, is applied to the device in thermal equilibrium. The current value after a time of 1500 fs is stored as a steady-state current, and then the same step voltage is applied to this steady-state value. This process is repeated until the external bias reaches its maximum value. Then the potential is decreased by applying a small negative bias to observe any hysteresis in the $I-V$ characteristics.

Figure 6 shows the $I-V$ characteristics of the device at room temperature. The $I-V$ characteristics of the device without a spacer layer (thicknesses of the highly doped ntype $\mathrm{GaAs}$ cathode and anode layers set to $24 \mathrm{~nm}$ ) are also shown in this figure. The peak voltage of the device with the spacer layers becomes larger than that without spacer layers because of the large voltage drop across them. The calculations in the negative differential resistance (NDR) regions are found to be slightly unstable compared with those for the positive differential resistance regions, and longer calculations were generally required to reach the steady state. Small intrinsic bistabilities in NDR regions are observed in both devices. In the case of the device with spacer layers it seems that, when bias is decreased, the $I-V$ curve in the NDR region shifts towards a lower voltage. Figure 7 shows energy band diagrams corresponding to the two stable states of the device

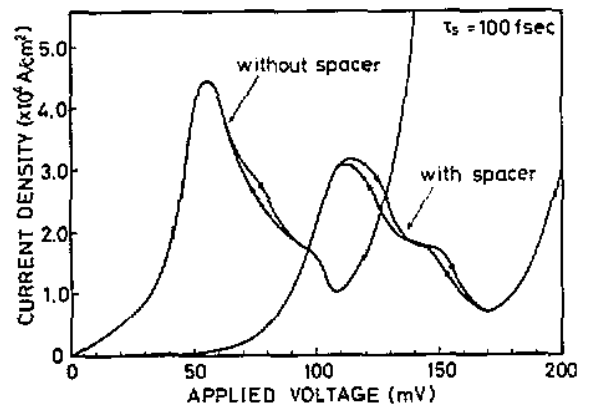

Figure 6. Current-voltage characteristics calculated for resonant tunnelling diodes with and without spacer layers. The applied bias is decreased after it reaches a maximum, to show the hysteresis. Bistability observed in the negative differential resistance region arises from dynamical electron redistribution in the quantum well.

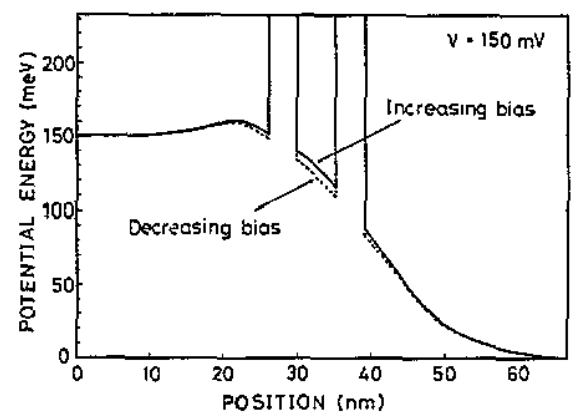

Figure 7. Self-consistent energy band diagrams calculated at two stable states under an applied bias of $0.15 \mathrm{~V}$. The upper curve corresponds to the larger current state, and the lower curve to the smaller current state.

for an external bias of $0.15 \mathrm{~V}$. When the bias is increasing, electrons with a sheet concentration of $2.1 \times 10^{11} \mathrm{~cm}^{-2}$ are accumulated in the quantum well at this bias. However, in the case of decreasing bias, the electron concentration goes down to $1.5 \times 10^{11} \mathrm{~cm}^{-2}$. The difference in the electron build-up leads to a different Hartree selfconsistent field, and the bottom of the quantum well in the case of increasing bias is almost $10 \mathrm{meV}$ higher than in the case of decreasing bias.

It should be noted that the energy band diagrams in figure 7 are vitually flat near the 
cathode and anode electrodes, and are similar to that obtained from screening theory, as a consequence of charge neutrality in these regions. This differs from the results from the transient Wigner function calculation [17] which led to a large potential drop in the cathode region because of heavy depletion of the Wigner distribution. Similar upward curving of the potential could be seen in the present calculation when a much larger value was assumed for the scattering relaxation time. Even though almost the same relaxation time is used, the resultant electron relaxation in the density matrix formula appears to be stronger than that in the Wigner function formula. This might be because of the different Ohmic contact model: the boundary condition (15) naturally conserves electron density at the cathode edge under an applied bias. Further analysis is being continued on this point by transforming the non-equilibrium density matrix obtained to the Wigner function, and will be reported elsewhere.

Another interesting feature in figure 6 is the current shoulder seen in the NDR regions. Similar structure is sometimes observed experimentally in samples with large $P / V$ current ratios, and several studies have been reported $[27,28]$ on extrinsic instability due to the bias circuit. From macroscopic circuit theory, it has been revealed that the $I-V$ characteristics of the RTD with a bias circuit have a current shoulder whose shape depends on $L C R$ parameters of the external circuit [28]. Because dynamics of electrons not only in the quantum well but also in the cathode and anode regions are now simulated exactly, the present results might show the existence of intrinsic instability of the system. We found that this current shoulder in the NDR region depends on the scattering relaxation time and generally vanishes with decreasing relaxation time. This fact implies that the structure is produced by the electrons undergoing coherent tunnelling and becomes small as the phase coherence of the electron waves degrades with increasing scattering. However, the microscopic physical mechanism causing this structure is not clear at present, and further investigation is necessary.

Figure $8(a)$ shows the $I-V$ characteristics of the device without spacer layers calculated with various values of relaxation time due to scattering: $\tau_{\mathrm{s}}=100 \mathrm{fs}$ (full curve); $80 \mathrm{fs}$ (broken curve); $60 \mathrm{fs}$ (one-dot chain curve); and $40 \mathrm{fs}$ (two-dot chain curve). As shown in this figure, the peak current decreases, and the valley current rapidly increases with a decrease in the relaxation time, resulting in a large degradation in the peak-tovalley current ratio. It should be noted that with a scattering time of $40 \mathrm{fs}$ the $I-V$ curve no longer has a NDR region. This strong dependence on $\tau_{\mathrm{s}}$ of the $P / V$ current ratio is
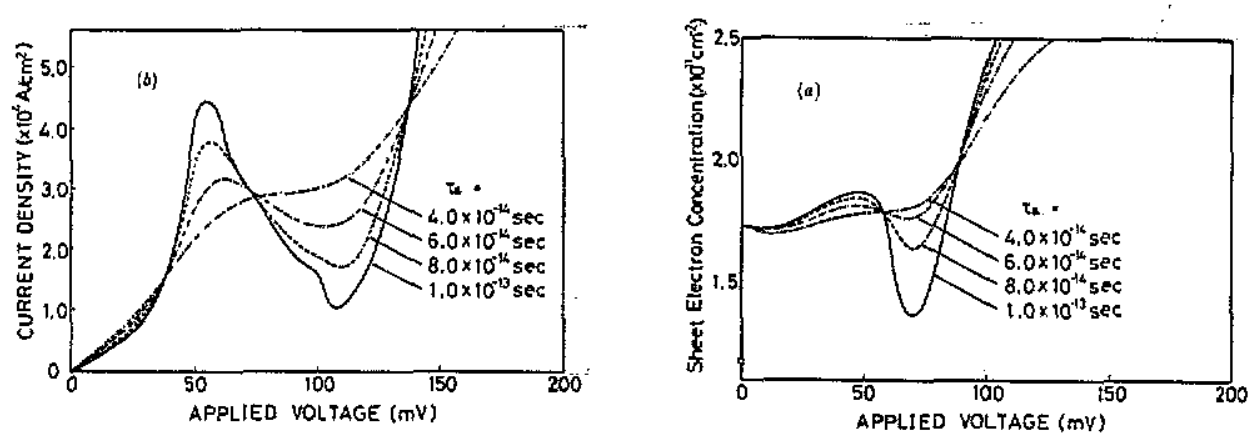

Figure 8. The applied voltage dependence of $(a)$ current density and $(b)$ sheet electron concentration in the quantum well calculated for the device without spacer layers with various values of $\tau_{\mathrm{s}}$. 

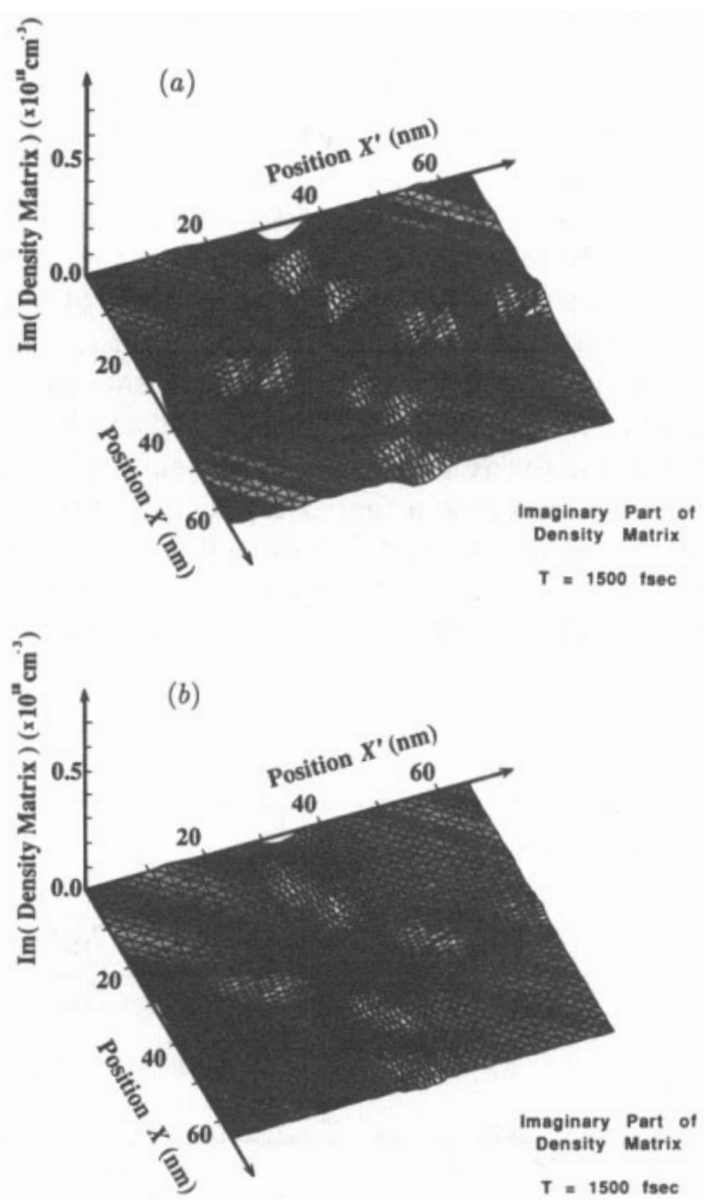

Figure 9. The imaginary part of the steady-state density matrix (at a time of $1500 \mathrm{fs}$ ) calculated with a $\tau_{\mathrm{s}}$ of $(a) 100 \mathrm{fs}$ and $(b) 40 \mathrm{fs}$. The reduced oscillatory behaviour of the off-diagonal elements in (b) signifies degradation of the phase coherence in the device due to a shorter scattering relaxation time.

understood as follows: figure $8(b)$ shows the voltage dependence of the sheet concentration, $n_{\mathrm{s}}$, of electrons accumulated in the quantum well calculated with various values of the relaxation time. It can be seen that the $n_{\mathrm{s}}-V$ curves have similar peak-to-valley structures corresponding to the NDR of the $I-V$ characteristics, and the peak-to-valley ratios also decrease with decreasing $\tau_{\mathrm{s}}$. Introducing a shorter relaxation time gives rise to more frequent momentum relaxation of electrons in the quantum well. In other words, the phase coherence of the electron waves at resonance degrades, and the tunnelling mechanism changes from coherent tunnelling to sequential tunnelling. The increase in $n_{\mathrm{s}}$ for the valley state indicates that a number of electrons are still present on the quasi-bound level even off-resonance, and these bring about a large valley current. Figure 9 shows the imaginary parts of the density matrix at the peak current state: calculated with $\tau_{\mathrm{s}}$ of $(a) 100 \mathrm{fs}$ and (b) $40 \mathrm{fs}$. As explained earlier, the phase coherence of the electron waves at resonance can be observed as the oscillatory behaviour of the 
off-diagonal elements. Comparing these two figures, it can be clearly seen that amplitude of the oscillation in figure $9(b)$ is much smaller than that in figure $9(a)$. These figures show us directly the degradation of the electron wave coherency due to scattering.

Finally, the calculated switching behaviours of the device with spacer layers are shown in figure 10. Figure 10(a) shows the current transient when the initial bias of $115 \mathrm{mV}$, corresponding to the peak voltage, is suddenly switched to $170 \mathrm{mV}$, corresponding to the valley voitage. The initial state at $t=0$ is a steady state under an applied bias of $115 \mathrm{mV}$, which was obtained as a result of continuous transient calculations with a small applied bias as described above (see the inset in figure $10(a)$ ). The meanings of the three curves in these figures are the same as in figure 5. Plasma current oscillation with the same period as in figure 5 is observed in the cathode and anode layers. The time dependence of the current density in the quantum well shows small oscillatory behaviour within some tens of femtoseconds. Because a similar rapid current transient has been observed in Wigner function calculations that neglect the self-consistent Hartree potential [16], this can be considered to arise from the rapid change in density of electrons accumulated in the quantum well after a sudden increase in applied voltage. The current
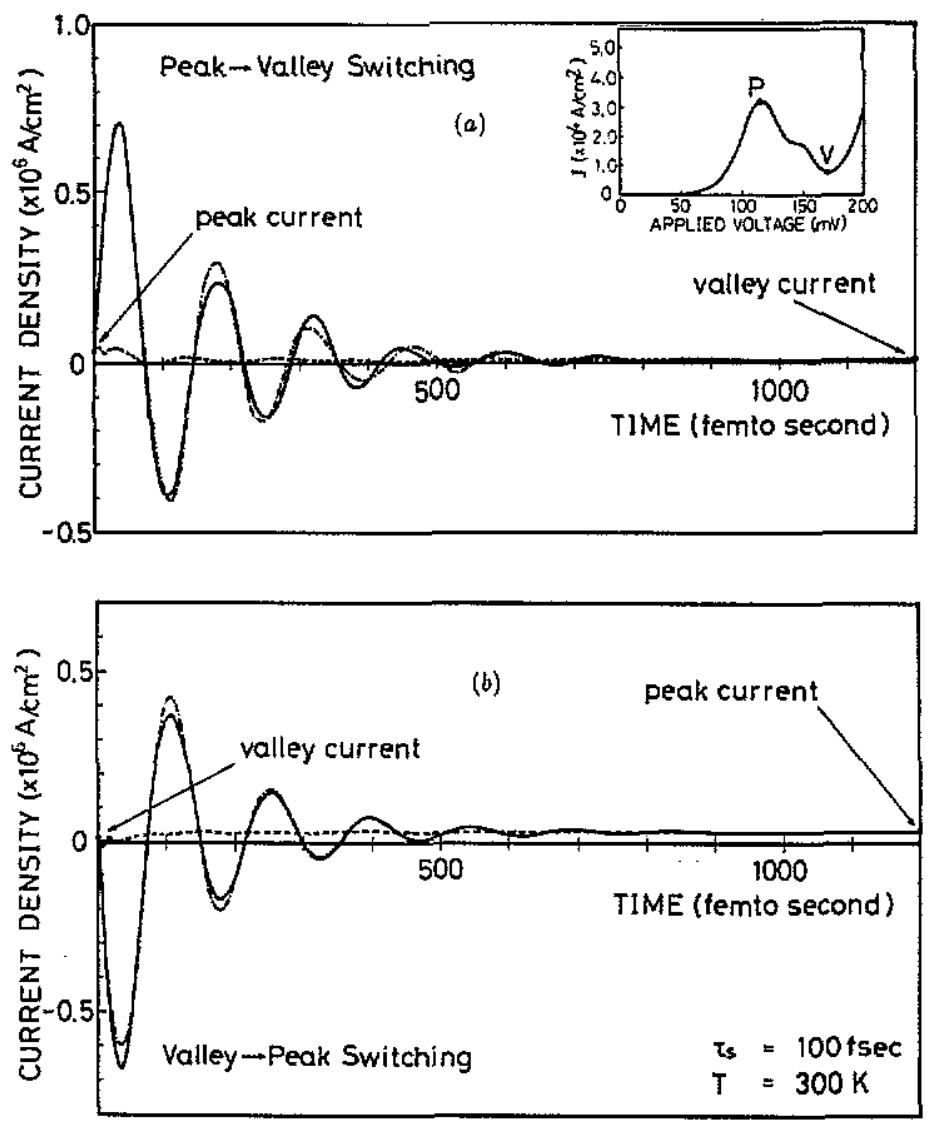

Figure 10. The time dependence of the current density for $(a)$ peak-to-valley and $(b)$ valleyto-peak switchings of the device with spacer layers (see the inset in $(a)$ ). 
transient for the reverse switching is shown in figure $10(b)$. The applied bias is suddenly switched from $170 \mathrm{mV}$ to $115 \mathrm{mV}$. The current density initially drops, and then oscillates with almost the same period as in $(a)$. For both peak-to-valley and valley-to-peak switchings, the device reaches the steady state at about one thousand femtoseconds, and the switching time is mainly determined by the plasma current oscillation which is a result of introducing the Hartree self-consistent field.

\section{Conclusion}

We have reported a new transient quantum device simulation based upon the statistical density matrix theory. The one-dimensional time-dependent Liouville-von Neumann equation for the electron density matrix was numerically solved by introducing the Hartree self-consistent field model for electron-electron interactions and the relaxation time approximation for scattering processes. The correct initial density matrix in thermal equilibrium was calculated by solving the Schrödinger equation and Poisson's equation self-consistently. By using this solution as an initial condition, the femtosecond time evolution of the density matrix under an applied voltage was determined by solving the density matrix equation implementing a Neumann-type boundary condition. The present simulation technology was successfully applied to the simple AlGaAs/GaAs resonant tunnelling diodes. Remarkable oscillatory behaviour was observed in the offdiagonal elements of the imaginary parts, which reveals the existence of the long-range phase correlations between quasi-bound electron waves in the quantum well and free electron waves in the cathode and anode layer. By utilizing this result, the peak-to-valley ratio of the $I-V$ characteristics was investigated from the viewpoint of phase coherence degradation due to scattering. The calculated $P / V$ current ratio degrades with decreasing scattering relaxation time. At the same time, it was clearly seen that amplitude of the off-diagonal oscillation decreases. These facts signify that the $P / V$ current ratio strongly depends on the phase coherence of the electron waves at the resonant state, and the calculated density matrix enables us to observe the degradation of the electron wave coherence due to scattering. Peak-to-valley and valley-to peak switching behaviours of the device were calculated. In both cases, the device reaches the steady state at a time of about $1000 \mathrm{fs}$, which is mainly determined by the plasma current oscillation in the cathode and anode layers. Intrinsic bistability of the $I-V$ characteristics was also analysed from the viewpoint of charge accumulation. Since a significant difference was found in the number of accumulated electrons at two stable states, it was clear that the current bistability observed in the NDR region results from electron redistribution in the quantum well. A current shoulder seen in the NDR region is also an interesting result which might show the existence of intrinsic instability of the device. Further investigation of its microscopic mechanism is still to be carried out.

\section{Acknowledgments}

The authors wish to thank Dr K Nakazato, Dr D Williams, Dr T Tanoue, Mr K Ka, Mr R Blaikie, and Dr J R A Cleaver for many helpful discussions, and Dr H Matsumura and $\mathrm{Dr} H$ Ahmed for their encouragement during the course of this work. 


\section{References}

[1] Tsu R and Esaki L 1973 Tunneling in a finite superlattice Appl. Phys. Lett. 22562

[2] Yokoyama N, Jmamura K, Muto S, Hiyamizu S and Nishi H 1985 A new functional, resonant tunneling hot electron transistor (RHET) Japan. J. Appl. Phys. 24 L853

[3] Seabaugh A C, Frensley W R, Randall J N, Reed M A, Farrington D L and Matyi RJ 1989 Pseudomorphic bipolar quantum resonant-tunneling transistor IEEE Trans. Electron Devices ED-36 23281988.

[4] Tanoue T, Mizuta $H$ and Takahashi S 1988 A triple-well resonant tunneling diode for multiple-valued logic application IEEE Electron Device Lett. EDL-9 365

[5] Datta S, Melloch MR, Bandyopadhyay S and Lundstron MS 1986 Proposed structure for a large quantum interference effects Appl. Phys. Lett. 48487

[6] Furuya $\mathrm{K}$ and Kurishima $\mathrm{K} 1988$ Electron wave diffraction by nanometer grating and its application for high-speed transistors J. Vac. Sci. Technol. B 61845

[7] Sols F, Macucci M, Ravaioli U and Hess K 1989 Theory of a quantum modulated transistor J. Appl. Phys. 663892

[8] Ohnishi H, Inata T, Muto S, Yokoyama N and Shibatomi A 1986 Self-consistent analysis of resonant turneling current Appl. Phys. Lett. 491248

[9] Mizuta H, Tanoue T and Takahashi S 1989 Theoretical analysis of peak-to-valley ratio degradation caused by scattering processes in multi-barrier resonant tunneling diodes IEEE/Cornell Conf. on Advanced Concepts in High Speed Semiconductor Devices and Circuits (Ithaca, NY: IEEE)

[10] Szafer A and Stone A D 1989 Theory of quantum conduction through a constriction Phys. Rev. Lett. 62 300

[11] Ter Haar D 1961 Theory and applications of the density matrix Rep. Prog. Phys. 24304

[12] Barker J R and Murray S1983 A quasi-classical formulation of the Wigner function approach to quantum ballistic transport Phys. Lett. 93A 271

[13] Xing D Y and Ting C S 1987 Green's-function approach to transient hot-electron transport in semiconductors under a uniform electric field Phys. Rev, B 353971

[14] Mason B A and Hess K 1989 Quantum Monte Carlo calculations of electron dynamics in dissipative solidstate sytems using real-time path integrals Phys. Rev. B 395051

[15] Ravaioli U, Osman A M, Poetz W, Kluksdahl N C and Ferry D K 1985 Investigation of baltistic transport through resonant-tunneling quantum wells using Wigner function approach Physica B 13436

[16] Frensley W R 1987 Wigner-function model of a resonant-tunneling semiconductor device Phys. Rev. B 36 1570

[17] Kluksdahl N C, Kriman A M, Ferry D K and Ringhofer C 1989 Self-consistent study of the resonanttunneling diode Phys. Rev. B 397720

[18] Frensley W R 1989 Quantum kinetic theory of nanoelectronic devices Nanostructure Physics and Fabrication, Proc. Int. Symp. (College Station, TX) (New York: Academic) p 231

[19] Frensley W R 1985 Simulation of resonant-tunneling heterostructure devices J. Vac. Sci. Technol. B 3 1261

[20] Brunetti R, Jacoboni C and Rossi F 1989 Quantum theory of transient transport in semiconductors: a Monte Carlo approach Phys. Rev. B 3910781

[21] Menziani P, Rossi F and Jacoboni C 1989 Impurity scattering in quantum transport simulation Solid State Electron. 321807

[22] Rossi $\mathrm{F}$ and Jacoboni 1989 A quantum description of drift velocity overshoot at high electric fields in semiconductors Solid State Electron. 321411

[23] Kriman A M, Kluksdahl N C and Ferry D K 1987 Scattering states and distribution functions for microstructures Phys. Rev. B 365953

[24] Levinson I B 1970 Translational invariance in uniform fields and the equation for the density matrix in the Wigner representation Sov. Phys.-JETP 30362

[25] Caldeira A O and Leggett A J 1983 Path integral approach to quantum Brownian motion Physica A 121 587

[26] Frensley W R 1990 Boundary conditions for open quantum systems driven far from equilibrium Rev. Mod. Phys. 62745

[27] Young J F, Wood B M, Liu H C, Buchanan M, Landheer D, SpringThorpe A J and Mandeville P 1988 Effect of circuit oscillations of the dc current-voltage characteristics of double barrier resonant tunneling structures Appl. Phys. Lett. 521398

[28] Belhadj CY, Martin K P, Ben Amor S, Rascol J J L, Potter R C, Hier H and Hemplling E 1990 Bias circuit effects on the current-voltage characteristic of double-barrier tunneling structures: experimental and theoretical results Appl. Phys. Lett. 5758 